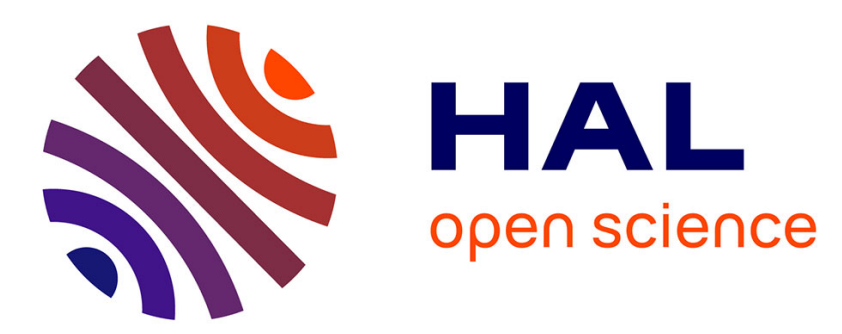

\title{
Ionization and recombination in nanosecond repetitively pulsed microplasmas in air at atmospheric pressure
}

\author{
Thomas Orrière, Eric Moreau, David Pai
}

\section{To cite this version:}

Thomas Orrière, Eric Moreau, David Pai. Ionization and recombination in nanosecond repetitively pulsed microplasmas in air at atmospheric pressure. Journal of Physics D: Applied Physics, 2018, 51 (49), pp.494002. 10.1088/1361-6463/aae134 . hal-03272472

\section{HAL Id: hal-03272472 \\ https://hal.science/hal-03272472}

Submitted on 28 Jun 2021

HAL is a multi-disciplinary open access archive for the deposit and dissemination of scientific research documents, whether they are published or not. The documents may come from teaching and research institutions in France or abroad, or from public or private research centers.
L'archive ouverte pluridisciplinaire HAL, est destinée au dépôt et à la diffusion de documents scientifiques de niveau recherche, publiés ou non, émanant des établissements d'enseignement et de recherche français ou étrangers, des laboratoires publics ou privés. 


\title{
Ionization and recombination in nanosecond repetitively pulsed microplasmas in air at atmospheric pressure
}

\author{
Thomas Orrière*, Eric Moreau, David Z. Pai*
}

Département Fluides, Thermique et Combustion, Institut Pprime (CNRS UPR 3346, Université de Poitiers, ENSMA), bd Marie et Pierre Curie, Téléport 2, BP 30179F, 86962 Chasseneuil Futuroscope

*Corresponding authors: thomas.orriere@univ-poitiers.fr, david.pai@univ-poitiers.fr

\begin{abstract}
We confine the nanosecond repetitively pulsed discharge (NRP) to the micrometer scale, in a $200-\mu \mathrm{m}$ discharge gap in air at atmospheric pressure and room temperature, focusing on measurements of the electron number density and electron temperature. The Stark broadening of $\mathrm{H}, \mathrm{O}$ and $\mathrm{N}$ atomic lines and electrical conductivity both show that the electron number density reaches a maximum value of $1 \times 10^{19} \mathrm{~cm}^{-3}$. Boltzmann plots show the electron temperature to be $72 \mathrm{kK}$ several nanoseconds after the end of the pulse of applied electric field. We will use these results to determine the mechanism responsible for electron loss during the early recombination phase $(t<500 \mathrm{~ns})$ and comment on the degree of ionization and dissociation.
\end{abstract}

\section{Introduction}

With nanosecond repetitively pulsed (NRP) discharges, it is generally possible to maintain higher average reduced electric fields than with dc, ac, or long pulsed excitation. As a result, the average electron kinetic energy is relatively high, and the degree of gas heating is reduced [1]. A high pulse repetition frequency $\left(F_{N R P}\right)$ enables the accumulation of active species and charge, leading to synergetic effects when $1 / F_{N R P}$ is shorter than the decay times of key species, such as electrons. Here, NRP discharges are defined by applied voltage pulse durations of $\sim 10 \mathrm{~ns}$ and pulse repetition frequencies of $1 \mathrm{kHz}$ or higher. We will refer to other pulsed discharges hereafter as 'nanosecond' discharges. 
Initial manuscript to Journal of Physics D: Special issue on chemical kinetics in non-equilibrium plasmas

The various regimes of NRP discharges generated in air at atmospheric pressure have found use in a wide range of applications. NRP dielectric barrier discharges (DBDs) have been studied for aerodynamic flow control [2], biomedical treatment [3], and ignition [4]. NRP glow discharges can improve the control of thermoacoustic instabilities [5]. Finally, NRP sparks have been explored for nanomaterials synthesis [6] and especially combustion [7]. The appropriate discharge for a given application depends on a number of parameters, the most important of which include the densities of charged and plasma chemical species.

The electron number density $\left(n_{e}\right)$ and recombination time $\left(\tau_{n e}\right)$ are key indicators of plasma density and vary greatly depending on the regime and experimental conditions. In air at atmospheric pressure, $n_{e}$ values of $10^{12}-10^{13} \mathrm{~cm}^{-3}$ have been measured for NRP glows [8,9] and DBDs [10]. NRP and nanosecond sparks can reach up to $n_{e}=10^{15} \mathrm{~cm}^{-3}$ [11-13]. When the gas temperature is high (e.g. $2000 \mathrm{~K})$, the electron recombination time of the NRP glow is $12 \mu$ due to the dissociative recombination to $\mathrm{NO}^{+}$, which is relatively slow $[8,9]$. When generated at $300 \mathrm{~K}$, the nanosecond spark recombination time is about $40 \mathrm{~ns}$ [11], which is likely faster due to electron attachment at low temperature.

We may ask whether the range of $n_{e}$ and/or $\tau_{n e}$ can be extended further for NRP discharges in air at atmospheric pressure, particularly when generated at room temperature. If so, then new possibilities for applications may include extending the post-discharge region to larger volumes. Three related discharges indicate that this should be possible. First, nanosecond discharges with long pulse duration (>100 ns) can reach up to $n_{e}=10^{17}-10^{19} \mathrm{~cm}^{-3}$ in $\mathrm{N}_{2}[14]$ and air $[15,16]$ at atmospheric pressure. In these discharges, however, high power dissipates over hundreds of nanoseconds, resulting in significant gas heating and low reduced electric field. Second, NRP discharges have been studied in the microplasma regime in noble gas mixtures at atmospheric pressure, where again $n_{e}=10^{16}-10^{18} \mathrm{~cm}^{-3}$ is attained [17-20]. In addition, $\tau_{n e}$ has been measured to be $100 \mathrm{~ns}$ or more [18-20]. Third, the millimeter-scale NRP spark generated in high-temperature $(475 \mathrm{~K})$ water vapor generates up to $n_{e}=3 \times 10^{18} \mathrm{~cm}^{-3}[21]$.

The aim of this study is thus to explore whether NRP microplasmas generated in air at atmospheric pressure and room temperature can attain similarly high electron number density and long recombination times as those in noble gas mixtures. We confine NRP microplasmas to $200 \mu \mathrm{m}$ in atmospheric air at $300 \mathrm{~K}$ in a pin-to-pin configuration and compare selected plasma properties to NRP spark discharges from previous studies in which larger gap distances were used. 
Initial manuscript to Journal of Physics D: Special issue on chemical kinetics in non-equilibrium plasmas

We will study the discharge by using electrical characterization and optical emission spectroscopy. In section 2, we will present the system for high-voltage pulse generation in the pin-to-pin configuration. Section 3 will concern the methods and hypotheses we use when determining the electron number density and temperature. In section 4, we will present and discuss our experimental results obtained mainly by Boltzmann plots, electrical conductivity, and the Stark broadening of atomic lines. Finally, in section 5 we will draw conclusions about our work.

\section{Experimental setup}

As shown on Figure 1, NRP microplasmas were generated by applying pulses of $2.5 \mathrm{kV}$ amplitude at $F_{N R P}=8 \mathrm{kHz}$ between two pure tungsten pin electrodes with radii of curvature of $280 \mu \mathrm{m}$, separated by a gap distance of $200 \mu \mathrm{m}$ in air at atmospheric pressure and room temperature. A generator (FID Technology FPG 40-30 NK) transmitted 30-ns high-voltage pulses through a $75-\Omega$ transmission line. Rough impedance matching was achieved by including two resistances of $200 \Omega$ and $100 \Omega$ at the load, resulting in $>80 \%$ energy transfer in all load conditions including the dynamic impedance of the microplasma. The voltage applied across the gap was measured by a passive probe (Lecroy PPE $6 \mathrm{kV}, 400 \mathrm{MHz}$ bandwidth), and the discharge current was measured by a current transformer (Bergoz CT-D5.0, $400 \mathrm{MHz}$ bandwidth). We recorded waveforms on an oscilloscope (Lecroy WaveRunner 204 MXI, 2 GHz bandwidth, 5 GS.s ${ }^{-1}$ ).

Optical emission spectroscopy (OES) was performed with a monochromator (Acton SP2500i) equipped with an intensified CCD (ICCD) camera (Princeton instrument PI-MAX 4), using a grating with 600 grooves $/ \mathrm{mm}$ blazed at $300 \mathrm{~nm}$. ICCD images were recorded via the spectrometer with the grating positioned to reflect light in zero order. Two off-axis parabolic mirrors with focal lengths of 5 and $20 \mathrm{~cm}$ formed the optical path. The total magnification was 4.4, and a diaphragm placed between the mirrors increased the depth of field to improve image sharpness. A single trigger source (Stanford Research Systems DG645) synchronized all devices. 
Initial manuscript to Journal of Physics D: Special issue on chemical kinetics in non-equilibrium plasmas

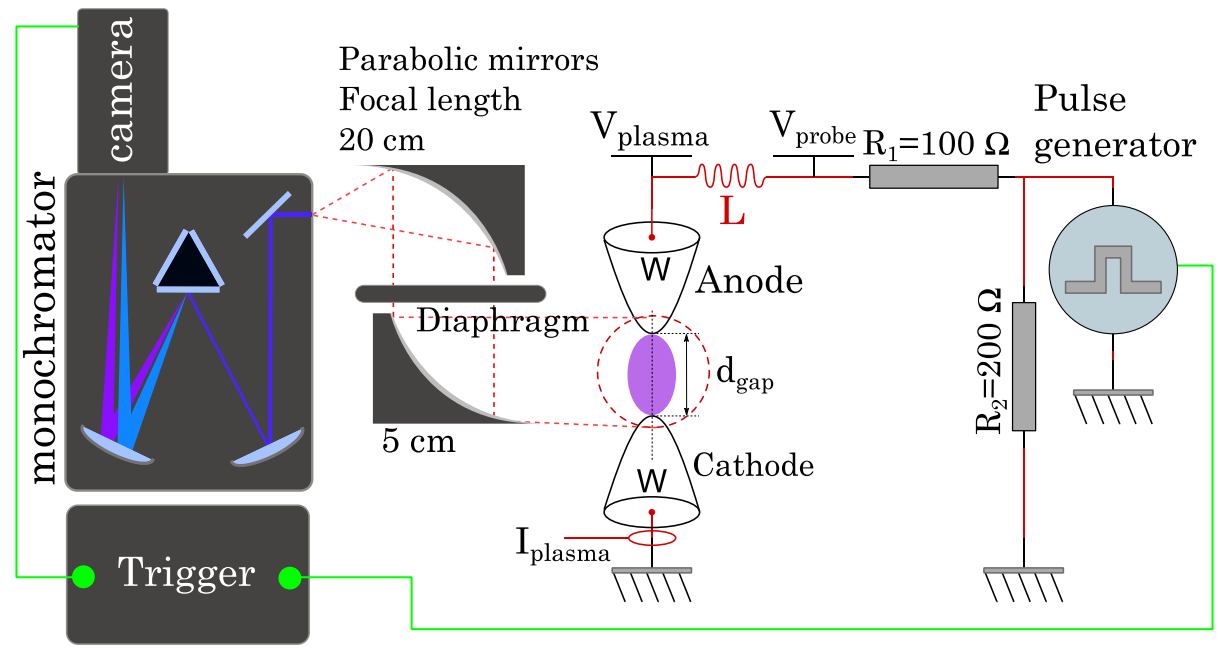

Figure 1: Schematic diagram of the discharge circuit and setup for optical emission spectroscopy.

\section{Experimental methods}

Here we explain key aspects of our experimental procedures. First, we account for parasitic elements in the discharge circuit when determining discharge voltage and current. The parasitic capacitance is negligible $(2.3 \mathrm{pF})$, so no correction is necessary for current waveforms. The parasitic inductance $L=180 \mathrm{nH}$ was determined by short-circuiting the electrodes together and scaling the induced voltage $L d I_{\text {plasma }} / d t$ to match the measured voltage waveform $V_{\text {probe }}$, as shown in Figure 2. This inductance value corresponds well to the approximate electrical length $(1 \mathrm{nH} / \mathrm{mm})$ [22] expected of the $23 \mathrm{~cm}$ of wire between the point of measurement by the voltage probe and the ground terminal. We used the short-circuit test to determine the delay between the current and the voltage probes, as demonstrated in Figure 2. All voltage waveforms are corrected for parasitic inductance to obtain the actual voltage across the gap $V_{\text {plasma }}=V_{\text {probe }}-$ $L d I_{\text {plasma }} / d t$. The energy calculated using the short-circuit test is $3 \pm 5 \mu \mathrm{J}$, which defines the detection limit of the discharge energy $U_{\text {plasma }}$. 
Initial manuscript to Journal of Physics D: Special issue on chemical kinetics in non-equilibrium plasmas

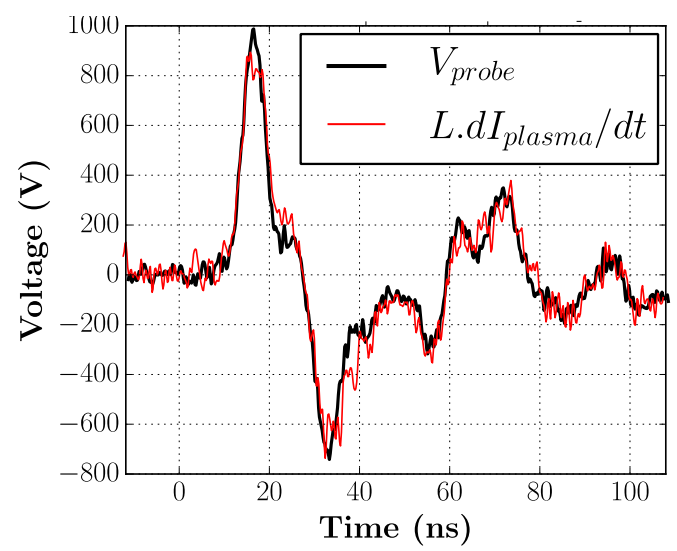

Figure 2: Measured voltage $\left(V_{\text {probe }}\right)$ and induced voltage calculated from measured current ( $\left.I_{\text {plasma }}\right)$, using the short-circuit test for parasitic inductance for the same high-voltage generator settings as in Figure 5.

Second, we account for electron-ion Coulomb interactions when determining the electron number density $n_{e}$ using corrected current-voltage waveforms according to equation (1), where $\sigma$ is the electrical conductivity, $e$ is the elementary charge, and $m_{e}$ is the electron mass. The electron collision frequency $v_{m}$ (equation (2)) includes terms for electron-neutral and electron-ion collisions with cross-sections $\sigma_{N}$ and $\sigma_{\text {coul }}$, respectively. The gas density is $N$, and the electron drift velocity is $v_{e}$. The volumetric rate $v_{e} \sigma_{c o u l}$ is calculated using equations (3) and (4) [23], where $\ln \Lambda$ is the Coulomb logarithm, $\varepsilon_{0}$ is the permittivity of free space, $k_{B}$ is the Boltzmann constant, and $T_{e}$ is the electron temperature.

$$
\begin{gathered}
\sigma=\frac{n_{e} e^{2}}{m_{e} v_{m}} \\
v_{m}=N v_{e} \sigma_{N}+n_{e} v_{e} \sigma_{\text {coul }} \\
v_{e} \sigma_{\text {coul }}=\frac{e^{4} \ln \Lambda}{9 \sqrt{2 m_{e}} \epsilon_{0}^{2}\left(\pi k_{B} T_{e}\right)^{3 / 2}} \\
\ln \Lambda=\ln \left(\frac{12 \pi\left(\epsilon_{0} k_{B} T_{e}\right)^{3 / 2}}{e^{3} n_{e}^{1 / 2}}\right)
\end{gathered}
$$

Equations (1) - (4) show that at high ionization fractions, the electron temperature must be accounted for when calculating $n_{e}$ because the electron-neutral and electron-ion collision frequencies vary as a function of $T_{e}$. To examine the influence of $T_{e}$, we use Bolsig+ [24] (Phelps 
Initial manuscript to Journal of Physics D: Special issue on chemical kinetics in non-equilibrium plasmas

database [25]), applying equation (3) to calculate the contribution of electron-neutral collisions to $v_{m}$. As shown in Figure 3, Coulomb interactions become dominant as $n_{e}$ approaches $10^{19} \mathrm{~cm}^{-3} \mathrm{but}$ remain minor at lower $n_{e}$, so long as $T_{e}$ is not too low.

Although Bolsig+ models steady-state conditions, its calculation of $T_{e}$ is reasonably close to those of dedicated models of pulsed discharges. With conditions $N=1,47 \times 10^{19} \mathrm{~cm}^{-3}, 100$ $\% \mathrm{~N}_{2}$ and gas temperature $T_{g}=500 \mathrm{~K}$, we obtain a mean electron temperature of $T_{e}=5,3 \mathrm{eV}$ using Bolsig+. In the same conditions but using a pulsed electric field, Colonna et al. [26] have calculated $T_{e}=6,3 \mathrm{eV}$ with a more complete set of cross-sections.

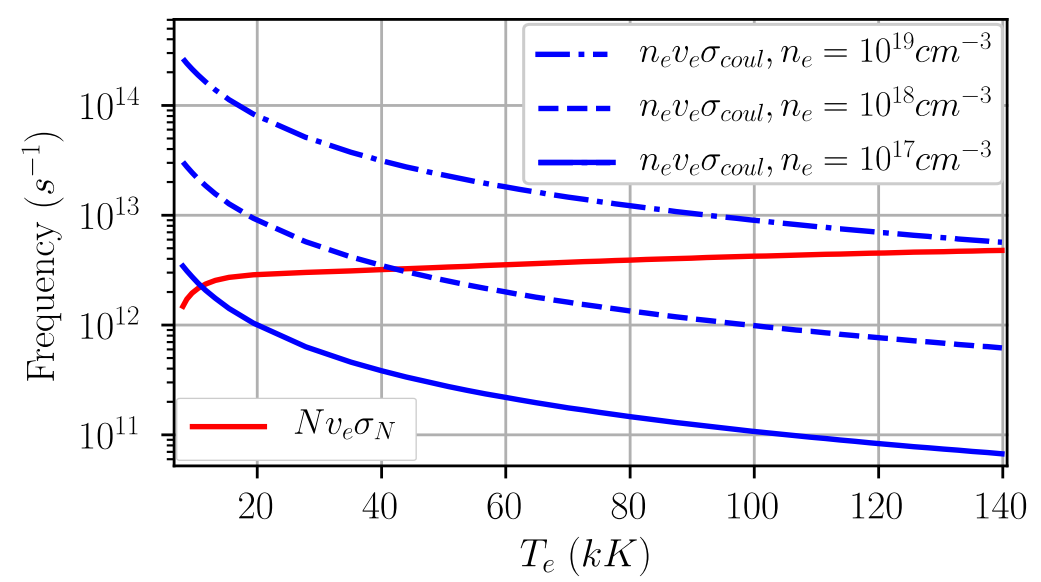

Figure 3: Electron-neutral (red) and electron-ion (blue) collision frequencies as a function of the electron temperature, calculated using Bolsig+ for the conditions of this work $(N=$ $1.98 \times 10^{19} \mathrm{~cm}^{-3}$, synthetic air with $80 \% \mathrm{~N}_{2}$ and $20 \% \mathrm{O}_{2}$ at $T_{g}=370 \mathrm{~K}$ ).

For a given experimental value of $\sigma$, the iterative procedure for solving equations (1) - (4) to find $n_{e}$ is as follows. First, we estimate $T_{e}$ using the approximate conditions of our study. For example, based on other measurements outside the scope of this work, we can estimate the initial values of the reduced electric field $E / N=600 \mathrm{Td}$ and gas temperature $T_{g}=370 \mathrm{~K}$ [27], thus yielding about $T_{e}=100 \mathrm{kK}$ according to Bolsig+. Such a high temperature is possible because, as we will see in section 4 , the electrons cool down to $T_{e}=70 \mathrm{kK}$ a few nanoseconds after the applied electric field decreases to zero.

We initially guess $n_{e}=2 \times 10^{19} \mathrm{~cm}^{-3}$ to fully calculate equation (2) via equations (3) and (4). A new value for $n_{e}$ is calculated using equation (1) and used to iterate through these equations again. We repeat this process until the value of $n_{e}$ converges. If the ionization fraction is low, 
Initial manuscript to Journal of Physics D: Special issue on chemical kinetics in non-equilibrium plasmas

then equation (2) reduces to $v_{m}=N v_{e} \sigma_{N}$, which is not especially sensitive to $T_{e}$ provided that $T_{e}>10 \mathrm{kK}$. If the ionization fraction is high, then there is some sensitivity to $T_{e}$. Because we cannot measure the electron temperature during the discharge phase, we include an uncertainty of $T_{e}=116 \pm 20 \mathrm{kK}$ when calculating equations (3) and (4).

Third, when evaluating the electron number density and electron temperature via OES, we fit the experimental spectra using different methods for modeling the continuum, molecular, and atomic spectral components. The superposition of modeled spectral components leads to accurate fits of the experimental spectra, as shown in Figure 4. The spectral range of measurement is small enough to approximate the continuum background as a straight line. The molecular bands of $\mathrm{N}_{2}$ (B-A) are modeled using Specair [28]. For neutral atoms and atomic ions, accurate fits are achieved using Voigt profiles, as shown in Figure 4. The spectral width $\left(\Delta \lambda_{\text {meas }}\right)$ of the fitted Voigt profiles are then used to determine the electron number density via the Stark broadening $\left(\Delta \lambda_{\text {stark }}\right)$ of the $\mathrm{H}_{\alpha}(656 \mathrm{~nm}), \mathrm{N}(746 \mathrm{~nm})$, and $\mathrm{O}(777 \mathrm{~nm})$ lines, according to equation (5). The Gaussian component of the profile is primarily due to instrumental broadening and is negligible. Thus, we consider only the Lorentzian components for the calculation of the electron density.

$$
\Delta \lambda_{\text {stark }}=\Delta \lambda_{\text {meas }}-\Delta \lambda_{v d w}-\Delta \lambda_{i}
$$

Table 1 summarizes the van der Waals $\left(\Delta \lambda_{v d w}\right)$ and instrumental $\left(\Delta \lambda_{i}\right)$ broadening for our case. To calculate $\Delta \lambda_{v d w}$, we use the maximum pressure of $7 \mathrm{~atm}$ expected to be produced by our discharge [27]. Doppler and resonant broadening components are negligible in our conditions [28]. Due to these other broadening mechanisms, our detection limit using the Stark broadening of $\mathrm{N}$ and $\mathrm{O}$ is $n_{e}=10^{17} \mathrm{~cm}^{-3}$.

Table 1: Spectral widths of instrumental and Doppler broadening.

\begin{tabular}{|c|c|c|c|}
\hline Lines & $\boldsymbol{N}(\mathbf{7 4 6} \mathbf{n m})$ & $\boldsymbol{O}(\mathbf{7 7 7} \mathbf{n m})$ & $\boldsymbol{H}_{\boldsymbol{\alpha}}$ \\
\hline $\boldsymbol{\Delta} \boldsymbol{\lambda}_{\boldsymbol{i}}(\mathbf{n m})$ & & $\mathbf{0 , 1 2}$ & \\
\hline $\boldsymbol{\Delta} \boldsymbol{\lambda}_{\boldsymbol{v} \boldsymbol{d} \boldsymbol{w}}(\mathbf{n m})$ & $2,0 \times P / T_{g a z}^{0,7}$ & $2,2 \times P / T_{g a z}^{0,7}$ & $5,41 \times P / T_{g a z}^{0,7}$ \\
\hline $\boldsymbol{\Delta} \boldsymbol{\lambda}_{\boldsymbol{v} \boldsymbol{d w}}(\mathbf{n m})$ & 0,12 & 0,13 & 0,31 \\
\hline references & {$[14]$} & {$[29]$} & {$[29]$} \\
\hline
\end{tabular}


Initial manuscript to Journal of Physics D: Special issue on chemical kinetics in non-equilibrium plasmas

For the Stark broadening of $\mathrm{H}_{\alpha}$, we calculate the electron number density from $\Delta \lambda_{\text {stark }}$ using equation (6) [30], which is a generalized result from the tabulated values provided by [31]. Temperature is accounted for via the modified reduced mass $\mu_{r}^{*}=\mu_{r} \times T_{e} / T_{g}$, where $\mu_{r}$ is the reduced mass of the emitter-perturber pair, expressed in amu. Formulas have been provided to fit the data from [31] but only for specific temperatures, for example $T_{e}=10 \mathrm{kK}$ in the case of [14]. We calculate the fitting parameters $\Delta \lambda_{0}$ and $\alpha_{\Delta \lambda}$ as a function of $\mu_{r}^{*}$, according to Table 2. We assume $\mu_{r}^{*}=10 \mathrm{amu}$, which is the largest value considered by [31]. The width can be expressed either as the full-width at half-maximum (FWHM) or the full-width at half-area (FWHA). As apparent from Table 2, the FWHA is less sensitive to temperature and will be used in this work for $\mathrm{H}_{\alpha}$, denoted as $\Delta \lambda_{\text {stark }-H_{\alpha}}$. Most importantly, the FWHA of $\mathrm{H}_{\alpha}$ is relatively insensitive to ion dynamics [31], which may become important at high ionization fraction.

$$
\Delta \lambda_{\text {stark- } H_{\alpha}}[\mathrm{nm}]=\Delta \lambda_{0}\left(\mathrm{n}_{e}\left[\mathrm{~cm}^{-3}\right] / 10^{17}\right)^{\alpha_{\Delta \lambda}}
$$

Table 2: Expressions for the fitting coefficients of equation (6).

\begin{tabular}{|c|c|c|}
\hline & $\boldsymbol{F W H M}$ & $\boldsymbol{F W H A}$ \\
\hline $\boldsymbol{\Delta} \boldsymbol{\lambda}_{\mathbf{0}}(\mathbf{n m})$ & $0,905 \times\left(\mu_{r}^{*} / 5\right)^{-0,0727}$ & $0,841 \times\left(\mu_{r}^{*} / 5\right)^{-0,149}$ \\
\hline $\boldsymbol{\alpha}_{\Delta \lambda}$ & $0,707 \times\left(\mu_{r}^{*}{ }_{5}\right)^{0,0246}$ & $0,694 \times\left(\mu_{r}^{*} / 5\right)^{0,0067}$ \\
\hline
\end{tabular}

However, since the widths of the broadening mechanisms are additive (equation (5)) only for the FWHM, we are obliged to calculate the FWHA in the following manner. First, we fit the experimental spectrum using a Voigt profile, and the width of the Lorentzian component is taken to be $\Delta \lambda_{\text {meas }}$ in equation (5). Then, the calculated value of $\Delta \lambda_{\text {stark }}$ is used to reconstruct a Lorentzian profile, whose FWHA is numerically determined and used as $\Delta \lambda_{s t a r k-H_{\alpha}}$ in equation (6). 
Initial manuscript to Journal of Physics D: Special issue on chemical kinetics in non-equilibrium plasmas
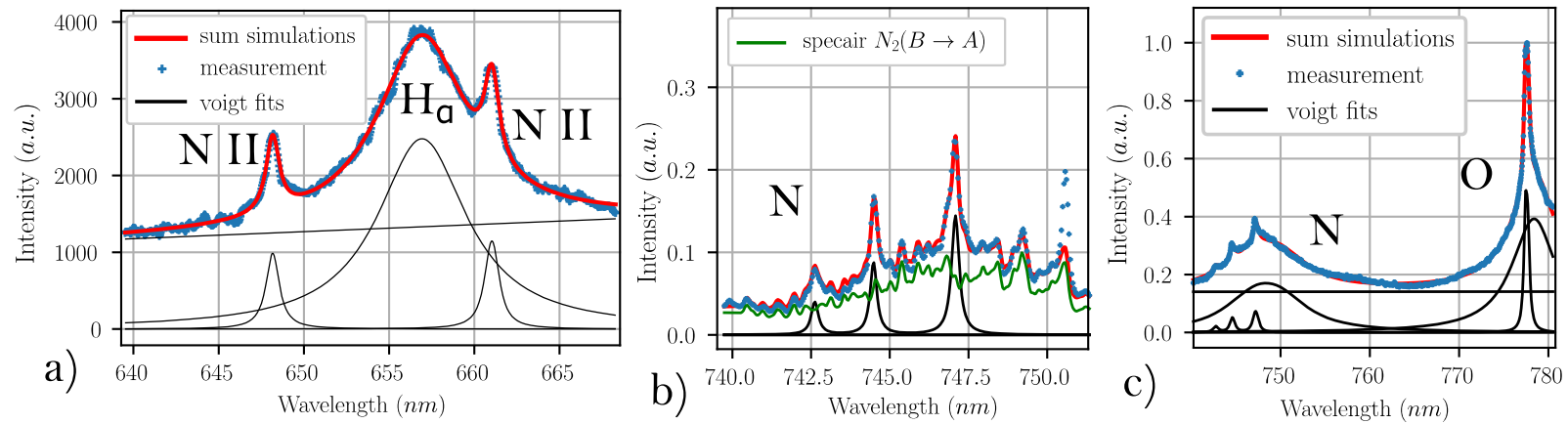

Figure 4: Examples of the agreement between simulated (lines) and experimental (symbols) spectra. Voigt profiles and straight lines are respectively used to model the atomic and continuum spectral components of a) $\mathrm{H}_{\alpha}$ and $\mathrm{N}^{+}$, b) $\mathrm{N}_{2}(\mathrm{~B}-\mathrm{A})$ and three $\mathrm{O}$ lines broadened by two different electron number densities at $t=5 \mathrm{~ns}$, and c) six lines each of $\mathrm{N}$ and $\mathrm{O}$ representative of two electron number densities at $t=8 \mathrm{~ns}$. Separate lines appear to merge into one when the spectral spacing between them is much smaller than the broadening.

For the Stark broadening of $\mathrm{N}$ and $\mathrm{O}$, we calculate $n_{e}$ from $\Delta \lambda_{\text {stark }}$ expressed as a FWHM using equation (7), taken from [32]. This is the width of a line profile found upon the convolution of the line profiles of electron-impact broadening and of the quasi-static quadratic Stark effect leading to ion broadening. It is thus a general representation of the total Stark broadening of nonhydrogenic neutral atoms. The effect of perturber ions on the broadening is accounted for by the parameter $\alpha_{e}\left(T_{e}\right)$. The parameter $R_{e}$ (equation (8)) accounts for the Debye shielding of perturber ions by free electrons, as well as ion-ion correlations.

$$
\begin{gathered}
\Delta \lambda_{\text {stark }}(\mathrm{nm})=2 \omega_{e}\left(T_{e}\right) \times\left[1+1,75\left(1-0.75 R_{e}\right) \alpha_{e}\left(T_{e}\right)\right] 10^{-16} n_{e} \\
R_{e}=8,99 \times 10^{-2} n_{e}^{1 / 6} T_{e}^{-1 / 2}
\end{gathered}
$$

For the lines of $\mathrm{N}$ and $\mathrm{O}$ of interest, we use tabulated values for the Stark width $\omega_{e}$ as well as $\alpha_{e}$ found on Table 4.5 of [32]. For $\mathrm{N}$, from $T_{e}=2.5$ to $80 \mathrm{kK}$ the values of $\omega_{e}$ and $\alpha_{e}$ vary from $2.99 \times 10^{-3}$ to $8.78 \times 10^{-3} \mathrm{~nm}$ and from $4.9 \times 10^{-3}$ to $2.2 \times 10^{-3} \mathrm{~nm}$, respectively. Recent calculations show similar sensitivity of the Stark width to temperature [33] and also validate the model used to derive equation (7) against experimental work published after [32]. For O, the variations of $\omega_{e}$ 
Initial manuscript to Journal of Physics D: Special issue on chemical kinetics in non-equilibrium plasmas

with $T_{e}$ are of similar magnitude. For our purposes, the overall sensitivity of equation (7) to electron temperature is low, as we will see in section 4.

Fourth, we equate $T_{e}$ with the excitation temperature $T_{e x c}$ of $\mathrm{N}^{+}$, determined by constructing Boltzmann plots of the available emission lines [32]. To distinguish closely spaced lines, we employ the fitting procedure described above. The intensity of each line is taken to be the peak value of its fitted Voigt profile. The condition $T_{e}=T_{\text {exc }}$ is satisfied for ions when the ionization fraction is high, such that electron-ion collisions dominant over other processes and equilibrium is achieved between the excited populations of ions and free electrons [32,34]. We will see in section 4 that the ionization fraction is indeed high when $\mathrm{N}^{+}$emission is present.

The same method as described above has been used by Lo et al. [15] to determine the electron temperature of a discharge similar to ours. The applied voltage pulse was longer than in our case, but $T_{e}$ was measured during the first 50 nanoseconds of the discharge. The evolution of $n_{e}$ and $T_{e}$ was demonstrated to be consistent with the thermal expansion of the discharge, thus supporting the validity of assuming $T_{e}=T_{\text {exc }}$. In addition, the $\mathrm{N}^{+}$energy transition selected are relatively close to the ionization potential energy of $\mathrm{N}^{+}$. It is known that the higher lying levels are in equilibrium with free electrons in wide range of conditions [35] p. 453.

Finally, we emphasize that measurements with a spatial resolution of $14 \mu \mathrm{m}$ along the discharge axis showed uniformity in electron number density and temperature. To improve the signal-to-noise ratio, we then integrated the signal over the entire discharge for all OES measurements shown in the following.

\section{Results and discussion}

The NRP microplasma under study is generated in open ambient air at $300 \mathrm{~K}$, in a reactor with a gap distance of $d_{g a p}=200 \mu \mathrm{m}$. The pulse repetition frequency is $F_{N R P}=8 \mathrm{kHz}$. The basic electrical and optical characteristics of the microplasma are shown in Figure 5. Although the pulse delivered by the generator is $30 \mathrm{~ns}$ in duration, the voltage applied over the discharge gap is only $10 \mathrm{~ns}$ due to breakdown. A maximum $V_{\text {plasma }}$ of $2.5 \mathrm{kV}$ falls over the discharge gap, corresponding to a reduced electric field of $600 \mathrm{Td}$, assuming that $N=1.98 \times 10^{19} \mathrm{~cm}^{-3}$ remains constant at the nanosecond time scale. The gas density is determined from the ideal gas law using $T_{g}=370 \mathrm{~K}$, which has been measured at $t=4 \mathrm{~ns}$ [27]. This temperature reflects the fact that, after a given pulse, the gas heats up but then nearly cools back down to the ambient temperature before the following pulse. 
Initial manuscript to Journal of Physics D: Special issue on chemical kinetics in non-equilibrium plasmas

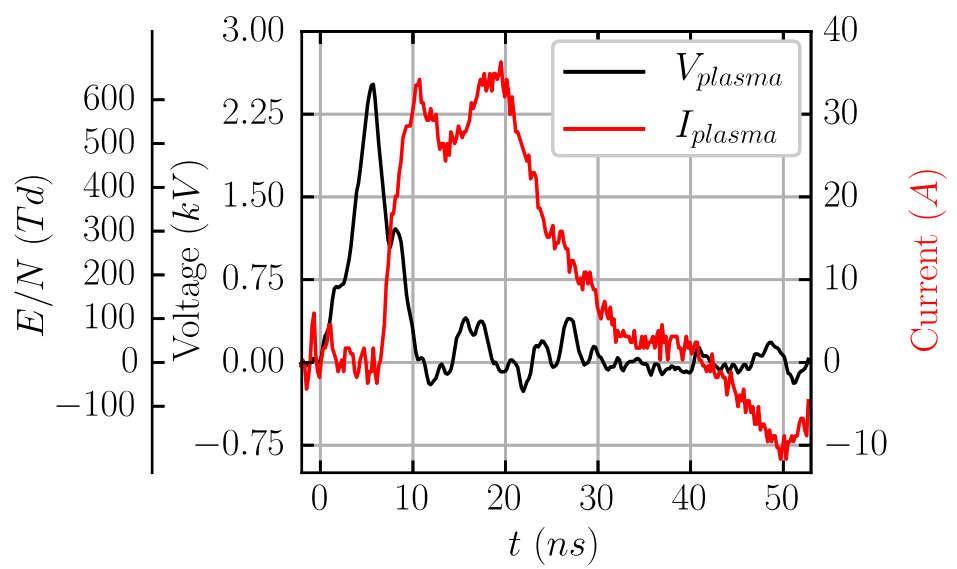

Figure 5: Measured single-shot voltage $\left(V_{\text {plasma }}\right)$ and current $\left(I_{\text {plasma }}\right)$ waveforms as a function of time for $F_{N R P}=8 \mathrm{kHz}$ and $d_{\text {gap }}=200 \mu \mathrm{m}$. The energy dissipated up to $t=45 \mathrm{~ns}$ is $U_{\text {plasma }}=$ $97 \mu$.

A spark-like breakdown begins at $t=7 \mathrm{~ns}$, when the applied voltage $V_{\text {plasma }}$ collapses to $0 \mathrm{~V}$ within $4 \mathrm{~ns}$, while the discharge current $I_{\text {plasma }}$ increases up to $36 \mathrm{~A}$. The double peak in the current waveform originates from the discharge. Oscillations from parasitic resonances in the rest of the discharge circuit can be discounted based on the short-circuit test described in section 3 , which shows purely resistive current (upon correcting $V_{\text {plasma }}$ for inductance) without any oscillations. The energy dissipated per pulse is $U_{\text {plasma }}=113 \pm 12 \mu \mathrm{J}$, and the peak dissipated power is $25 \mathrm{~kW}$.

Using current-voltage waveforms such as those shown in Figure 5, we can calculate the plasma impedance for $t>7 \mathrm{~ns}$, when there is measurable current. The plasma radius during this time is $r_{p}=20 \pm 4 \mu \mathrm{m}$ [27]. Thus, the current density is on the order of $10^{6} \mathrm{~A} / \mathrm{cm}^{2}$, which is arclike. Assuming that the voltage drop over the cathode fall region is also arc-like and thus lower than that of a normal glow in air $(\sim 300 \mathrm{~V})$, we can approximate the plasma impedance as a pure resistance $R_{\text {plasma }}$. The electrical conductivity can now be calculated as $\sigma=d_{\text {gap }} / \pi r_{p}^{2} R_{\text {plasma }}$, and equations (1) - (4) can be used to determine the electron number density for the brief interval when both current and voltage are measurable. Figure 6 shows that $n_{e}$ increases from about $10^{15} \mathrm{~cm}^{-3}$ up to a maximum of $1 \times 10^{19} \mathrm{~cm}^{-3}$ by $t=13 \mathrm{~ns}$. As stated above, $N=1.98 \times 10^{19} \mathrm{~cm}^{-3}$, yielding an ionization fraction of 0.5 in terms of the original gas before dissociation. 

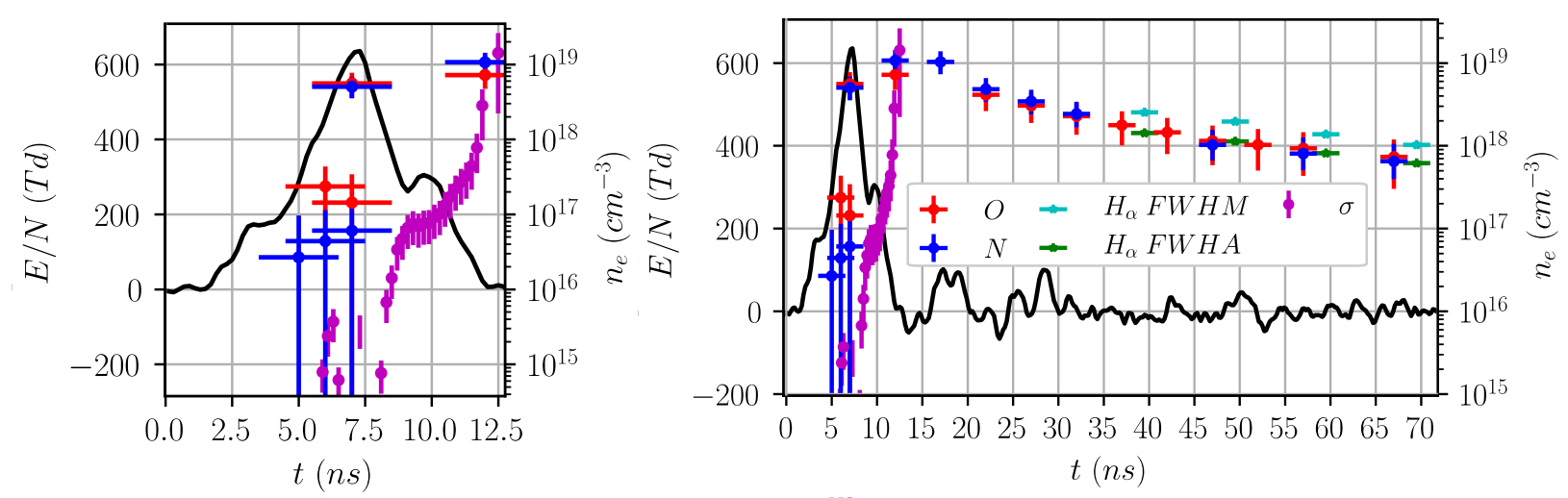

Figure 6: Measured electron number density during the high-voltage pulse (left) and early post-discharge (right) phases, determined from the electrical conductivity and the Stark broadening of $\mathrm{H}, \mathrm{O}$, and $\mathrm{N}$ atoms (symbols). For reference, the average reduced electric field (lines) is also shown.

The maximum value of $n_{e}$ determined from $\sigma$ agrees well with that obtained from the Stark broadening of $\mathrm{O}$ and $\mathrm{N}$, also shown in Figure 6. The data shown are calculated for $T_{e}=20 \mathrm{kK}$, and the uncertainty is bounded by the cases for $T_{e}=10 \mathrm{kK}$ and $80 \mathrm{kK}$. This range covers the expected variation of $T_{e}$ during the early post-discharge phase, according to measurements to be presented shortly. However, at $t<8 \mathrm{~ns}$ Stark broadening does not give a definitive measurement. This is because the gate width of the camera is limited to $3 \mathrm{~ns}$, and the jitter of the spark-like breakdown is about $1 \mathrm{~ns}$. The time resolution is therefore insufficient to capture the abrupt change in $n_{e}$ that is apparent from current-voltage measurements. Indeed, Figure 4 (right) shows that both narrow and broad spectra, corresponding respectively to low and high $n_{e}$, are acquired together during this time. Such mixed spectra have been observed previously by [20,21].

Starting at $t=40 \mathrm{~ns}$, the $\mathrm{H}_{\alpha}$ line becomes available for determining electron number density. In Figure 6, we show $n_{e}$ determined from the FWHA using equation (6), as well as the FWHM for direct comparison with previous work [14]. We see that there is agreement between the various Stark broadening measurements $\left(\mathrm{O}, \mathrm{N}, \mathrm{FWHA}\right.$ of $\left.\mathrm{H}_{\alpha}\right)$ at $t>35 \mathrm{~ns}$. Our combined measurements for the electron number density are thus mutually consistent for $t>12 \mathrm{~ns}$.

The high $n_{e}$ measured for NRP microplasmas is found more commonly in atmospheric nanosecond discharges generated in millimeter-scale gaps but with much longer pulse 
Initial manuscript to Journal of Physics D: Special issue on chemical kinetics in non-equilibrium plasmas

durations. Horst et al. [14]measured $n_{e} \approx 5 \times 10^{18} \mathrm{~cm}^{-3}$ for a discharge in $\mathrm{N}_{2}$ with small amounts of $\mathrm{H}_{2} \mathrm{O}$, where $d_{\text {gap }}=2 \mathrm{~mm}$, and the pulse width is $250 \mathrm{~ns}$. Lo et al. [15] found $n_{e}=9.2 \times 10^{18} \mathrm{~cm}^{-3}$ for a discharge in air with $d_{g a p}=3 \mathrm{~mm}$ and a pulse duration of $700 \mathrm{~ns}$. It is also possible to obtain such high densities in dielectric barrier discharges (DBDs), for example at higher pressure [36,37]. On the other hand, for millimeter-scale gaps and pulse durations similar to ours, $n_{e}$ is generally much lower. For NRP spark discharges, Pai et al. and Rusterholtz et al. [12,13] measured $n_{e}=2-3 \times 10^{15} \mathrm{~cm}^{-3}$ generated in similar experimental conditions except that $d_{\text {gap }}=4-5 \mathrm{~mm}$ and the initial $T_{g}=1500 \mathrm{~K}$ due to preheating of the air. Compared to the NRP microplasma, the millimeter-scale NRP spark produces similar current amplitudes but possesses much larger discharge diameters $(450-700 \mu \mathrm{m})$.

The high ionization fraction of NRP microplasmas results in the strong presence of atomic ion lines $\left(\mathrm{O}^{+}\right.$and $\left.\mathrm{N}^{+}\right)$in the OES spectra during the post-discharge phase, suggesting their involvement in electron recombination. Supposing high densities of $\mathrm{O}^{+}$and $\mathrm{N}^{+}$, recombination is likely to proceed via three-body recombination (R1), where $A$ represents either $\mathrm{O}$ or N. Molecular ions may remain present, particularly $\mathrm{N}_{2}{ }^{+}$and perhaps $\mathrm{NO}^{+}$as well, the latter being the primary ion involved in recombination at high temperature $\left(T_{g}=2000 \mathrm{~K}\right)$ [9]. These ions should recombine with electrons via dissociative recombination, reactions (R2) and (R3).

$$
\begin{array}{cc}
A^{+}+e+e \rightarrow A+e & k_{3 C}=0.7 \times 10^{-19}\left(300 / T_{e}\right)^{4.5} \mathrm{~cm}^{6} \mathrm{~s}^{-1} \\
N_{2}^{+}+e \rightarrow N+N & k_{R D-N 2}=3 \times 10^{-7}\left(300 / T_{e}\right)^{0.5} \mathrm{~cm}^{3} \mathrm{~s}^{-1} \\
N O^{+}+e \rightarrow N+O & k_{R D-N O}=4 \times 10^{-7}\left(300 / T_{e}\right)^{1.5} \mathrm{~cm}^{3} \mathrm{~s}^{-1}
\end{array}
$$

If we assume that the ion number density $n_{+}$is equal to $n_{e}$, and that $T_{e}$ is constant, then (R1), (R2) and (R3) can be separately solved for the electron number density as a function of time, respectively yielding equations (9) and (10). We calculate starting at time $t=t_{0}=12 \mathrm{~ns}$ with the measured initial electron number density $n_{e 0}=n_{e}(t=12 \mathrm{~ns})=1 \times 10^{19} \mathrm{~cm}^{-3}$.

$$
\frac{d n_{e}(t)}{d t}=-k_{3 C} n_{e}^{3} \rightarrow n_{e}(t)=\frac{n_{e 0}}{\sqrt{1+2 k_{3 C} n_{e 0}^{2}\left(t-t_{0}\right)}}
$$


Initial manuscript to Journal of Physics D: Special issue on chemical kinetics in non-equilibrium plasmas

$$
\frac{d n_{e}(t)}{d t}=-k_{R D} n_{e}^{2} \rightarrow n_{e}(t)=\frac{n_{e 0}}{1+k_{R D} n_{e 0}\left(t-t_{0}\right)}
$$

This model of electron recombination (equations (9) and (10)) is compared to the experimental data in Figure 7. Even when assuming a high $T_{e}=80 \mathrm{kK}$, the dissociative recombination of $\mathrm{N}_{2}{ }^{+}$and $\mathrm{NO}^{+}$is clearly too fast compared to experiment. A lower value of $T_{e}$ would exacerbate this problem. However, three-body recombination of atomic ions demonstrates much better agreement, as the experimental decay falls between the curves modeled using $T_{e}=40$ and $80 \mathrm{kK}$, which we will see are reasonable values at these times. Thus, we see that the relatively slow recombination in the early post-discharge phase can be explained if the electron temperature is kept high.

Horst et al. [14] also measured both a high electron number density and a slow decay in a 2-mm discharge gap in $\mathrm{N}_{2}$ but with a longer high-voltage pulse (250 ns). Thus, slow decay is not exclusive to $\mathrm{N}_{2} / \mathrm{O}_{2}$ mixtures and can also occur in pure $\mathrm{N}_{2}$. Judging their electron temperature to be low $(10 \mathrm{kK})$, Horst et al. suggested that a signification production of electrons via Penning ionization of the excited states $\mathrm{N}^{*}$ and $\mathrm{N}_{2} *$ could be responsible for the slow decay. High densities of these excited states could be generated by the recombination of $\mathrm{N}^{+}$with an electron. We will see that $T_{e}$ in our case should be higher. We do not consider heavy ions such as $\mathrm{N}_{4}{ }^{+}$, formed by the Penning ionization of $\mathrm{N}_{2} *$, because we do not observe any $\mathrm{N}_{2} *$ via OES in the recombination phase. Furthermore, we expect metastable $\mathrm{N}_{2}$ to be quenched by $\mathrm{O}_{2}$ [40]. In the following, we will show that very high levels of ionization and dissociation coupled with a high $T_{e}$ can explain the slow decay via reaction (R1), even at times later than shown in Figure 7. 
Initial manuscript to Journal of Physics D: Special issue on chemical kinetics in non-equilibrium plasmas

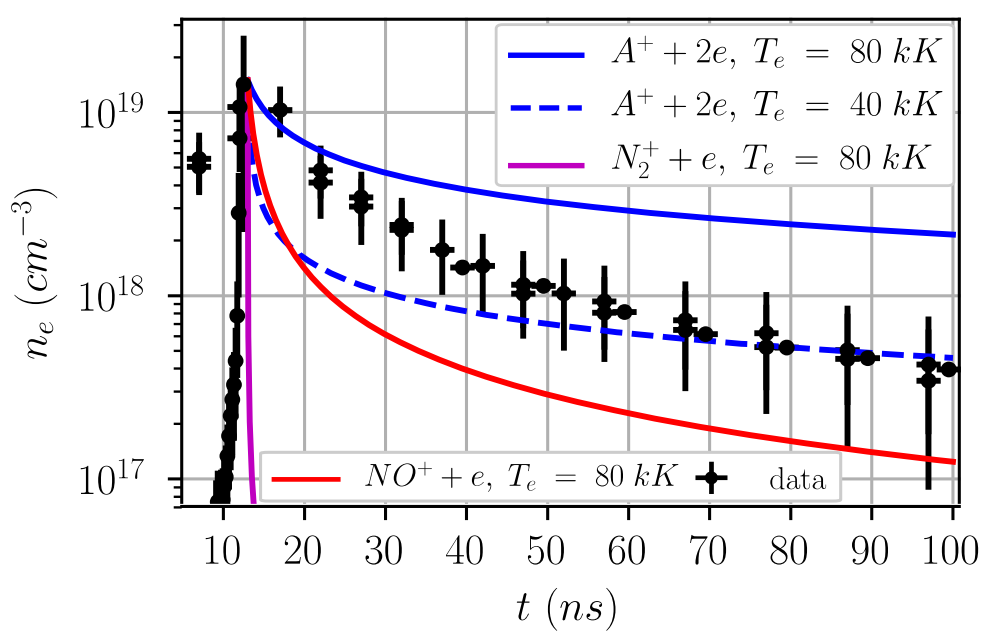

Figure 7: Comparison of modeled (lines, equations (9) and (10)) and experimental electron number density (symbols, taken from Figure 6, excluding the FWHM of $\mathrm{H}_{\alpha}$ ) during the early post-discharge phase.

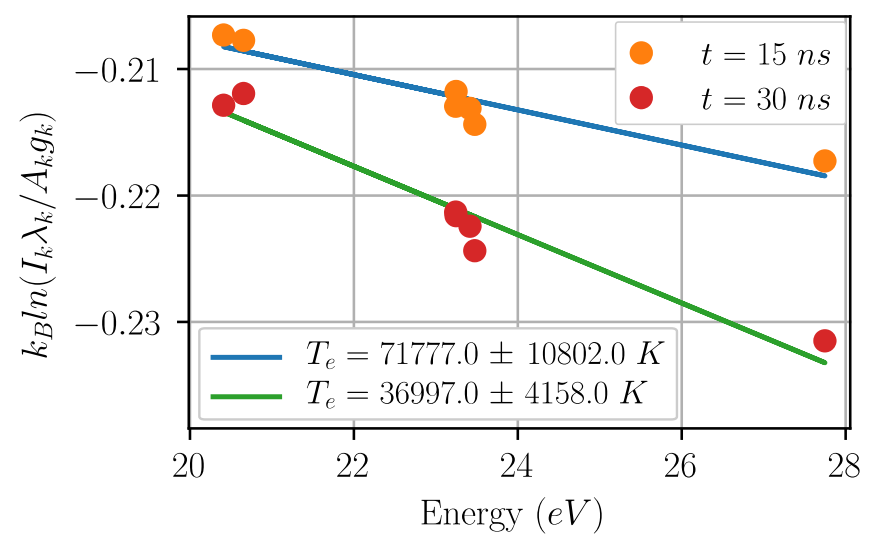

Figure 8: Boltzmann plots (lines) constructed from the measured emission intensities of $\mathrm{N}^{+}$ lines (symbols), at $t=15$ and $30 \mathrm{~ns}$.

There is indeed experimental evidence for high electron temperature, as shown in Figure 8. The Boltzmann plots of $\mathrm{N}^{+}$at $t=15$ and $30 \mathrm{~ns}$ correspond to $T_{e}=72$ and $37 \mathrm{kK}$, respectively. The cooling rate is thus $0.2 \mathrm{eV} / \mathrm{ns}$, which is noticeably slower than the rate of $5 \mathrm{eV} / \mathrm{ns}$ predicted by the model of [26], although their model is for $\mathrm{N}_{2}$ rather than air. Lo et al. [15] measured $T_{e}=42 \mathrm{kK}$ up to $40 \mathrm{~ns}$, but as mentioned in section 1 the current was maintained for a longer time than in our case due to a longer duration of the voltage pulse, thus prolonging electron heating. The most 
Initial manuscript to Journal of Physics D: Special issue on chemical kinetics in non-equilibrium plasmas

interesting comparison is with a nanosecond plasma generated in neon at 0.53 atm [19], where $T_{e}=25 \mathrm{kK}$ was directly measured using Thomson scattering at $15 \mathrm{~ns}$ into the post-discharge phase. It thus appears that $T_{e}$ can remain high even after electron heating ceases (i.e. no applied field) if a noble gas is used. In this case, the electrons cannot dissipate energy into vibrational modes.

The difference in electron cooling between molecular and atomic gases can be illustrated by considering the mean free paths of different types of collisions. The mean free path of electronneutral collisions is $\lambda_{N}=1 / N \sigma_{N}$, where $\sigma_{N}$ is the cross-section for electron-neutral momentum transfer. The characteristic length of $T_{e}$ relaxation is $\lambda_{T_{e}}=\lambda_{N} / \sqrt{\delta}$. For a dissociated gas, the electron energy loss coefficient is $\delta=2 m_{e} / m_{0}$, where $m_{0}$ is the mass of the atom. On the other hand, for a molecular gas $\delta \approx 100$ [35], leading to a much smaller $\lambda_{T_{e}}$ than for atoms. It is possible that our NRP microplasma finds itself in the state of an atomic gas, if the air becomes mostly dissociated.

If an atomic gas is furthermore strongly ionized, then other mechanisms of electron heating and cooling enter into consideration. In particular, electrons can lose energy via electron-ion collisions, but this mechanism of cooling is mitigated for three reasons. First, the ions are progressively removed due to recombination. In a molecular gas with low ionization fraction (such as that modeled by Colonna), the electron-neutral collision frequency does not decrease for this reason. Second, heating via super-elastic collisions counteracts cooling via electron-ion collisions. Finally and most importantly, the electron energy loss coefficient is not significantly different between a neutral atom or an atomic ion [23] (p 17). Thus, the energy lost per collision with an atomic ion is at least $10^{5}$ less than with a molecule. According to Figure 3, even at $T_{e} \sim 10 \mathrm{kK}$ and $n_{e}=10^{19} \mathrm{~cm}^{-3}$, the electron-ion collision frequency is only about 100 times greater than the electron-neutral collision frequency. Thus, the increased collision frequency with atomic ions cannot compensate for the low electron energy loss compared to molecules.

We return now to our model of recombination, reinforced by evidence of slow electron cooling and a mostly atomized gas. We suppose that $T_{e}=T_{e 0} \exp \left(-\left(t-t_{0}\right) / \tau_{T_{e}}\right)$, where $T_{e 0}$ is the initial value of the electron temperature, and $\tau_{T e}$ is its decay time. Reaction $(\mathrm{R} 1)$ can now be modeled with a time-varying reaction coefficient $k_{3 C}(t)$, yielding the improved rate equation (11), with constants $k_{A}=0.7 \times 10^{-19} \times\left(300 / T_{e 0}\right)^{4.5}$ and $\tau_{A}=\tau_{T_{e}} / 4.5$. The solution for $n_{e}$ is equation (12), calculated starting at time $t=t_{0}=30 \mathrm{~ns}$ with the measured initial electron number density 
Initial manuscript to Journal of Physics D: Special issue on chemical kinetics in non-equilibrium plasmas

$n_{e}(t=12 \mathrm{~ns})=n_{e 0}=3 \times 10^{18} \mathrm{~cm}^{-3}$. The result is compared in Figure 9 against experimental data determined from the Stark broadening of $\mathrm{H}_{\alpha}$, whose emission persists longer in the post-discharge phase $(t<500 \mathrm{~ns})$. The best agreement is obtained using the values $T_{e 0}=44 \mathrm{kK}$ and $\tau_{T e}=186 \mathrm{~ns}$. This value of $T_{e 0}$ is close to the measured value of $37 \mathrm{kK}$ at $t=30 \mathrm{~ns}$, which is also shown in Figure 9.

$$
\begin{gathered}
\frac{d n_{e}}{d t}=-k_{A} n_{e}^{3} \exp \left(\frac{t-t_{0}}{\tau_{A}}\right) \\
n_{e}(t)=\frac{n_{e 0}}{\sqrt{1+2 k_{A} \tau_{A} n_{e 0}^{2}\left(\exp \left(\left(t-t_{0}\right) / \tau_{A}\right)-1\right)}}
\end{gathered}
$$

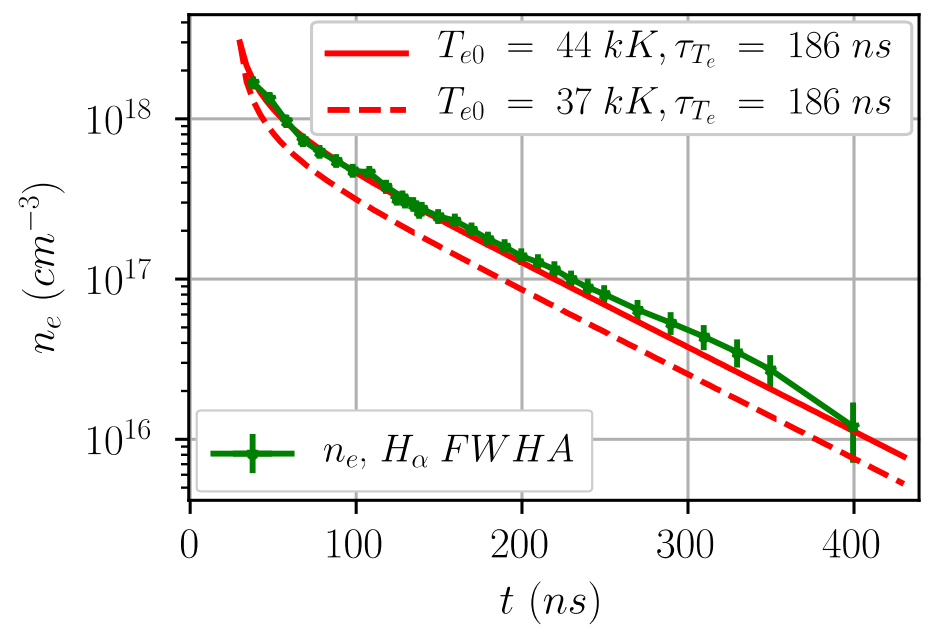

Figure 9: Comparison of measured electron number density (symbols) obtained via the Stark broadening of $\mathrm{H}_{\alpha}$ and the improved model (equation (12)) of recombination (lines).

Finally, the conditions of our discharge warrant the consideration of alternative mechanisms of electron generation during the recombination phase. Penning associative ionization of $\mathrm{O}$ or $\mathrm{N}$ atoms is not likely to be significant during the recombination phase because we do not observe excited $\mathrm{N}_{2}$ or $\mathrm{N}_{2}{ }^{+}$, and neutral-neutral collisions are much less frequent than electron-ion collisions. Furthermore, the rate of associative ionization would have to overcome that of its reverse process, namely the fast recombination processes R2 and R3 (Figure 7). The most probable reaction would be $\mathrm{R} 4$ with excited atoms $\mathrm{O}^{*}$ and $\mathrm{N}^{*}$ (see [41] and [42]): 
Initial manuscript to Journal of Physics D: Special issue on chemical kinetics in non-equilibrium plasmas

$$
O^{*}+N^{*} \rightarrow N^{+}+e \quad k_{N O} \approx 2 \times 10^{-11} \mathrm{~cm}^{3} \mathrm{~s}^{-1}\left(T_{\text {gas }}=300 \mathrm{~K}\right)
$$

Experimentally, the emission intensities from the excited states of $\mathrm{N}$ and $\mathrm{O}$ both decrease with the same decay time as $n_{e}$ [27]. Therefore the mutual ratios between $\mathrm{N}^{*}, \mathrm{O}^{*}$ and $n_{e}$ should remain about constant during recombination. We can then compare the production and loss of electrons by comparing the rate coefficients $k_{N O}$ and $k_{R D-N O}$. At $t=30 \mathrm{~ns}$, recombination proceeds with $k_{R D-N O}=3 \times 10^{-10} \mathrm{~cm}^{3} \mathrm{~s}^{-1}$, which will only increase with time as $T_{e}$ decreases. On the other hand, $k_{N O}$ is much smaller and is not very sensitive to the gas temperature. We thus expect R3 to be faster than $\mathrm{R} 4$, such that associative ionization is unlikely to slow down net recombination by generating electrons during the post-discharge phase. Finally, the ground states of $\mathrm{N}$ and $\mathrm{O}$ can also participate in thermal associative ionization, but this process requires a threshold energy of $2.8 \mathrm{eV}$ [43] (p. 385). The required gas temperature for such high kinetic energies is higher than what we expect from this type of discharge [44]. However, the reaction R4 with its rate presented by Popov in [41] only take in account the ground state of $\mathrm{O}$ and $\mathrm{N}\left({ }^{2} \mathrm{P}\right)$. It follows that the production of $\mathrm{NO}^{+}$have been underestimate due to the presence of other excited atoms. Therefore, $\mathrm{NO}^{+}$could be dominant, especially for $t>100 \mathrm{~ns}$ when the density of $\mathrm{N}^{*}$ and $\mathrm{O}^{*}$ should be very high.

We turn to previous experimental work to evaluate further whether $\mathrm{N}^{+}$or $\mathrm{NO}^{+}$is the main recombining ion. If $\mathrm{NO}^{+}$were the main recombining ion in hot air (which is likely in our case [27]), then the recombination time should be on the order of tens of microseconds, as demonstrated experimentally for NRP glow discharges by Packan[9]. As discussed earlier, Horst [14] observed a similar recombination time as shown in Figure 9, except in $\mathrm{N}_{2}$. The absence of oxygen suggests that a nitrogen species such as $\mathrm{N}^{+}$enables a strongly ionized plasma in hot air to recombine relatively quickly.

Electron-impact stepwise ionization may be significant because of the high temperature of the remaining electrons and a high density of excited $\mathrm{O}$ and $\mathrm{N}$. The characteristic time of threebody recombination is $\tau_{n e}$ and varies from $4 \mathrm{~ns}$ to $90 \mathrm{~ns}$ in the first $100 \mathrm{~ns}$ of our measurement. The characteristic time of ionization from energy level $n$ with population $N_{n}$ is $\tau_{e-n}=1 /\left(N_{n} \times k_{e-n}\right)$. If ionization is to overtake recombination, we must have $\tau_{e-n}>\tau_{n e}$. This situation is only possible via stepwise ionization for $t<100 \mathrm{~ns}$, if we assume densities of excited $\mathrm{N}$ or $\mathrm{O}$ are on the order of $10^{19} \mathrm{~cm}^{-3}$. Using the cross sections of the excited states of $\mathrm{N}\left({ }^{2} \mathrm{D}^{\circ}\right)$ or $\mathrm{N}\left({ }^{2} \mathrm{P}^{\circ}\right)$ [45] yields $k_{e-n} \sim$ 
Initial manuscript to Journal of Physics D: Special issue on chemical kinetics in non-equilibrium plasmas

$10^{-11} \mathrm{~cm}^{3} \cdot \mathrm{s}^{-1}$ for $T_{e}>30000 \mathrm{~K}$. Considering the ground state of N, with our estimation of $T_{e}$ and cross-sections from Wang et al. [45], we find $k_{e-n}<10^{-12} \mathrm{~cm}^{3} \cdot \mathrm{s}^{-1}$ using a Boltzmann solver $\left(\mathrm{BOLOS}^{2}\right.$ based on Bolsig+ [24]). Thus, only stepwise ionization may enter into consideration in

the early stages of the recombination phase $(t<100 \mathrm{~ns})$, and only upon assuming the maximum possible density of excited atoms. The possible result would be a lower initial temperature $T_{e} 0$ and time constant $\tau_{\mathrm{Te}}$.

\section{Conclusion}

We have characterized NRP microplasmas confined in a $200-\mu \mathrm{m}$ gap generated in air at atmospheric pressure and room temperature. Both Stark broadening and electrical conductivity measurements agreed that the electron number density reaches a maximum value of $1 \times 10^{19} \mathrm{~cm}^{-3}$, corresponding to an ionization fraction of air of 0.5 . At atmospheric pressure, $n_{e}$ values reaching $10^{19} \mathrm{~cm}^{-3}$ have been previously reported for millimeter-scale nanosecond discharges of much longer pulse duration [15]. In our case, short ( 30 ns) pulses and a micrometric gap are used.

After reaching its maximum value, $n_{e}$ decays relatively slowly with a recombination time of $90.8 \mathrm{~ns}$. This is consistent with a slow relaxation of $T_{e}$ and the nearly complete ionization and dissociation of the gas in the microplasma. The electron temperature is measured to be $72 \mathrm{kK}$ at $5 \mathrm{~ns}$ after the end of the voltage pulse. It is possible that the maximum value of $T_{e}$ is reached beforehand, while the electrons are still being heated by the applied electric field.

We modeled the electron recombination using a single reaction: three-body recombination. There is a strong coherence between the model and the measurements of electron number density and electron temperature. The best agreement of the model with experiment shown in Figure 9 is for an initial value of $T_{e}$ that is within $20 \%$ the experimental value of $37 \mathrm{kK}$. We also used experimental values of $n_{e}$ as initial values for the model. The only true free parameter in the model is $\tau_{T e}=186 \mathrm{~ns}$. However, this relaxation time is much longer than would be expected in a molecular gas [26] and is consistent with our experimental evidence of slow electron cooling due to a very high degree of dissociation. We also considered the possible role of ionization processes during the recombination phase, in particular associative and stepwise ionization stemming from the possibly high densities of excited $\mathrm{O}$ and $\mathrm{N}$ atoms.

\footnotetext{
${ }^{2}$ https://github.com/aluque/bolos
} 
Initial manuscript to Journal of Physics D: Special issue on chemical kinetics in non-equilibrium plasmas

Compared to its closest counterpart, the millimeter-scale NRP spark discharge, the NRP microplasma produces higher $n_{e}$ and $T_{e}$ throughout its volume. In future work, we plan to investigate the discharge dynamics, as well as other thermal, chemical kinetic, and electrohydrodynamic phenomena related to this NRP microplasma. In particular, we aim to study the electron kinetics during the post-discharge phase to improve the model of recombination. In particular, we neglected the production of ions and the presence of $\mathrm{NO}^{+}$during the post-discharge phase. Those aspects should be investigated to fully explain $n_{e}$ decay in the early afterglow.

\section{Acknowledgements}

Thomas Orrière has been supported by a $\mathrm{PhD}$ fellowship from the Ministère de l'Education Nationale, de 1'Enseignement Supérieur et de la Recherche. We also gratefully acknowledge financial support from the Agence Nationale de la Recherche programs JCJC PLASMAFACE (ANR-15-CE06-0007-01) and INOPLAS (ANR-13-ASTR-0015-01), the "Investissements d'Avenir" program LABEX INTERACTIFS (ANR-11-LABX-0017-01) of the French government, the CPER program of the Poitou-Charentes region, and the European Union (FEDER/FSE/FEADER programs).

\section{References}

[1] P. K. Chu, editor, Low Temperature Plasma Technology: Methods and Applications (CRC Press, Boca Raton, 2014).

[2] K. D. Bayoda, N. Benard, and E. Moreau, J. Appl. Phys. 118, 063301 (2015).

[3] H. Ayan, D. Staack, G. Fridman, A. Gutsol, Y. Mukhin, A. Starikovskii, A. Fridman, and G. Friedman, J. Phys. Appl. Phys. 42, 125202 (2009).

[4] S. A. Shcherbanev, S. A. Stepanyan, N. A. Popov, and S. M. Starikovskaia, Phil Trans R Soc A 373, $20140342(2015)$.

[5] D. A. Lacoste, Y. Xiong, J. P. Moeck, S. H. Chung, W. L. Roberts, and M. S. Cha, Proc. Combust. Inst. 36, 4183 (2017).

[6] D. Z. Pai, K. (Ken) Ostrikov, S. Kumar, D. A. Lacoste, I. Levchenko, and C. O. Laux, Sci. Rep. 3, (2013).

[7] D. A. Xu, M. N. Shneider, D. A. Lacoste, and C. O. Laux, J. Phys. Appl. Phys. 47, 235202 (2014).

[8] C. H. Kruger, C. O. Laux, L. Yu, D. M. Packan, and L. Pierrot, Pure Appl. Chem. 74, 337 (2002).

[9] D. Packan, Repetitive Nanosecond Glow Discharge in Atmospheric Pressure Air / (2003).

[10] J. L. Walsh and M. G. Kong, Appl. Phys. Lett. 91, 251504 (2007).

[11] F. Grisch, G.-A. Grandin, D. Messina, and B. Attal-Trétout, Comptes Rendus Mécanique 337, 504 (2009).

[12] D. Z. Pai, D. A. Lacoste, and C. O. Laux, Plasma Sources Sci. Technol. 19, 065015 (2010). 
Initial manuscript to Journal of Physics D: Special issue on chemical kinetics in non-equilibrium plasmas

[13] D. L. Rusterholtz, D. A. Lacoste, G. D. Stancu, D. Z. Pai, and C. O. Laux, J. Phys. Appl. Phys. 46, 464010 (2013).

[14] R. M. van der Horst, T. Verreycken, E. M. van Veldhuizen, and P. J. Bruggeman, J. Phys. Appl. Phys. 45, 345201 (2012).

[15] A. Lo, A. Cessou, C. Lacour, B. Lecordier, P. Boubert, D. A. Xu, C. O. Laux, and P. Vervisch, Plasma Sources Sci. Technol. 26, 045012 (2017).

[16] M. Janda, V. Martišovitš, K. Hensel, L. Dvonč, and Z. Machala, Plasma Sources Sci. Technol. 23, 065016 (2014).

[17] B.-D. Huang, X.-M. Zhu, K. Takashima, and Y.-K. Pu, J. Phys. Appl. Phys. 46, 464011 (2013).

[18] B. Du, N. Sadeghi, T. V. Tsankov, D. Luggenhölscher, and U. Czarnetzki, Plasma Sources Sci. Technol. 21, 045015 (2012).

[19] N. Bolouki, K. Tomita, S. Hassaballa, Y. Yamagata, and K. Uchino, Jpn. J. Appl. Phys. 54, $016101(2015)$.

[20] X.-M. Zhu, J. L. Walsh, W.-C. Chen, and Y.-K. Pu, J. Phys. Appl. Phys. 45, 295201 (2012).

[21] F. Sainct, Etude de la réactivité de décharges électriques nanoseconde à la pression atmosphérique dans la vapeur d'eau (Ecole Centrale Paris, 2014).

[22] A. V. Mezhiba and E. G. Friedman, Power Distribution Networks in High Speed Integrated Circuits (Springer Science \& Business Media, 2004).

[23] Y. P. Raizer, Gas Discharge Physics (Springer, 1992).

[24] G. J. M. Hagelaar and L. C. Pitchford, Plasma Sources Sci. Technol. 14, 722 (2005).

[25] Lxcat, Phelps Database, https://fr.lxcat.net/data/set_specA.php, n.d.

[26] G. Colonna, V. Laporta, R. Celiberto, M. Capitelli, and J. Tennyson, Plasma Sources Sci. Technol. 24, 035004 (2015).

[27] T. Orriere, Confinement Micrométrique de Décharges Pulsées Nanosecondes Dans L'air à Pression Atmosphérique et Effets électro-Aérodynamiques (Poitiers, 2018).

[28] C. O. Laux, T. G. Spence, C. H. Kruger, and R. N. Zare, Plasma Sources Sci. Technol. 12, 125 (2003).

[29] C. O. Laux, Optical Diagnostics and Radiative Emission of Air Plasmas (Stanford, 1993).

[30] N. Dardaine, Estimation de La Densité électronique Dans Les Plasmas Froids D’air à Pression Atmosphérique Grâce à L'effet Stark (Université de Poitiers, Poitiers, 2017), p. 15.

[31] M. A. Gigosos, M. Á. González, and V. Cardeñoso, Spectrochim. Acta Part B At. Spectrosc. 58, 1489 (2003).

[32] H. R. Griem, Plasma Spectroscopy (McGraw-Hill, 1964).

[33] Y. Liu, W. Huo, A. Wray, and D. Carbon, in 43rd AIAA Pap. 2012-2739 Thermophys. Conf. (American Institute of Aeronautics and Astronautics, 2012).

[34] C. A. Bye and A. Scheeline, Appl. Spectrosc. 47, 2022 (1993).

[35] M. Mitchner and C. H. Kruger, Partially Ionized Gases (1973).

[36] Shcherbanev, Filamentary Nanosecond Surface Dielectric Barrier Discharge at Elevated Pressures. Streamer-to-Filamentary Transition and Application for Plasma Assisted Combustion. (Ecole Polytechnique, 2016).

[37] S. A. Stepanyan, M. A. Bouhmedi, G. Vanhove, P. Desgroux, N. Popov, and S. Starikovskaya, in 52nd Aerosp. Sci. Meet. (American Institute of Aeronautics and Astronautics, 2014).

[38] P. M. Capitelli, P. C. M. Ferreira, P. B. F. Gordiets, and P. A. I. Osipov, in Plasma Kinet. Atmospheric Gases (Springer Berlin Heidelberg, 2000), pp. 119-154. 
Initial manuscript to Journal of Physics D: Special issue on chemical kinetics in non-equilibrium plasmas

[39] Gen. Phys. Inst., Moscow, Russia, I. A. Kossyi, A. Y. Kostinsky, A. A. Matveyev, and V. P. Silakov, Plasma Sources Sci. Technol. 1, 207 (1992).

[40] G. D. Stancu, M. Janda, F. Kaddouri, D. A. Lacoste, and C. O. Laux, (2009).

[41] N. A. Popov, Plasma Phys. Rep. 35, 436 (2009).

[42] N. D. Lepikhin, A. V. Klochko, N. A. Popov, and S. M. Starikovskaia, Plasma Sources Sci. Technol. 25, 045003 (2016).

[43] Y. B. Zel'dovich and Y. P. Raizer, in Phys. Shock Waves High-Temp. Hydrodyn. Phenom. (Academic Press, 1967), p. ix.

[44] A. Lo, A. Cessou, P. Boubert, and P. Vervisch, J. Phys. Appl. Phys. 47, 115201 (2014).

[45] Y. Wang, O. Zatsarinny, and K. Bartschat, Phys. Rev. A 89, 062714 (2014). 